\title{
PESQUISA E DESENVOLVIMENTO DE NOVAS FORMAS FARMACÊUTICAS PEDIÁTRICAS NO TRATAMENTO DE DOENÇAS NEGLIGENCIADAS
}

\author{
L. P. SIQUEIRA ${ }^{1}$, J.F.B. RODRIGUES ${ }^{1}$, E. O. SILVA $^{1}$, V. A. W. SALES ${ }^{1}$, I. N. G. \\ BARBOSA $^{1}$, C. G. SILVA ${ }^{1}$, L. C. P. B. B. SILVA ${ }^{1}$, A. L. M. D. SOUSA ${ }^{1}$, L. C. \\ CARDOSO $^{1}$, L. A. ROLIM ${ }^{2}$, R.M.F. SILVA ${ }^{1}$, P.J. ROLIM NETO ${ }^{1}$
}

${ }^{1}$ Universidade Federal de Pernambuco, Centro de Ciências da Saúde, Departamento de Ciências farmacêuticas, Laboratório de Tecnologia dos Medicamentos

${ }^{2}$ Universidade do Vale de São Francisco, Central de Análise de Fármacos, Medicamentos e Alimentos

E-mail para contato: jamillyfernanda_@hotmail.com

RESUMO - Doenças tropicais negligenciadas são um grupo de doenças transmissíveis, cujo tratamento é precário, não havendo muitos investimentos principalmente pelo fato dessas enfermidades afetarem a população mais carente. A porção pediátrica da população é considerada como grupo de risco para essas afecções, sendo necessário o desenvolvimento de formulações que atendam essa fração populacional. O objetivo do referido trabalho foi fazer uma revisão bibliográfica sobre investimentos na pesquisa e desenvolvimento de formulações pediátricas no tratamento de doenças negligenciadas, a partir de um levantamento de dados, realizado em sites periódicos, incluindo como base o Science Direct, Scielo e Google acadêmico, no periodo de 2009 a 2017. O desenvolvimento de medicamentos pediátricos tem sido estimulado por recentes legislações, resultando em esforços significativos para desenvolver formulações adequadas à idade aceitáveis para uso em pacientes pediátricos. As iniciativas de política pediátrica fizeram progresso significativo no avanço de um entendimento global de que a avaliação de novos medicamentos deve incluir formulações que atendam às necessidades pediátricas. Ainda assim, há uma necessidade urgente em disponibilizar novos medicamentos, pois os medicamentos utilizados contra doenças infecciosas correm o risco de desenvolver resistência. Além disso, há necessidade de novos medicamentos com regimes mais simples e que tenham melhor tolerabilidade e perfis de segurança.

Palavras-chave: Doenças negligenciadas, pediatria, indústria farmacêutica.

ABSTRACT - Tropical neglected diseases are a group of transmissible diseases whose treatment is precarious, and there are no much investments because these illnesses affect the most deprived population. The pediatric portion of the population is considered a risk group for these affections, so it is necessary the development of formulations that meet this population fraction. The objective of this work was to make a literature review about the investment in research and development of pediatric formulations for neglected diseases treatment, from a data survey, carried out in periodic websites, including as bases the Science Direct, Scielo, and Google Scholar, from 2009 to 2017. The development of pediatric medicines has been encouraged by recent legislations, resulting in significant efforts to develop acceptable age- 
appropriate formulations to be used in pediatric patients. Pediatric policy initiatives have made a significant progress in advancing a global understanding that the evaluation of new drugs must include formulations that meet pediatric needs. Even so, there is an urgent need to make new drugs available, as drugs used against infected diseases are at risk of developing resistance. In addition, there is the need for new drugs with simpler regimens and better tolerability and safety profiles.

Keywords: Neglected diseases, pediatrics, pharmaceutical industry.

\section{INTRODUÇÃO}

Doenças tropicais negligenciadas são um grupo de afecções transmissíveis, em sua maioria causada por protozoários e helmintos na transmissão por vetores. O tratamento para esse grupo de agravos é precário e desatualizado por parte da indústria farmacêutica, não há muitos investimentos principalmente pelo fato dessas enfermidades afetarem a população mais carente (OLIVEIRA, 2009). Dentro dessa realidade, a fração da população considerada como grupo de risco para essas tais doenças ainda são mulheres grávidas e crianças, especialmente por suas particularidades farmacocinéticas e farmacodinâmicas, sendo necessário o desenvolvimento de formulações que atendam e deem o suporte necessário para uma terapia otimizada com eficácia e segurança para essa população. Em seu desenvolvimento farmacotécnico, o medicamento dificilmente passa por estudos com o objetivo de atingir uma formulação de cunho pediátrico, com dosagem específica e adequada para a capacidade da criança, sendo necessário adequar a melhor forma farmacêutica (EMA, 2013; WELLS, 2017).

Em particular, há poucos estudos são descritos na literatura quanto ao desenvolvimento farmacotécnico de novas formulações pediátricas no tocante às doenças classificadas como negligenciadas, estimulando a medicina pediátrica a usar a forma de prescrição "off-label", o que pode trazer mais um agravo para a saúde do paciente ao se deparar com problemas relacionados à medicamentos (ELDER; HOLM; KUENTZ, 2017).

\section{MATERIAIS E MÉTODOS}

A construção do referido trabalho, foi embasada, a partir de um levantamento de dados, realizado em sites periódicos, incluindo como base o Science Direct, Scielo e Google acadêmico, no período de 2009 a 2017. A pesquisa por palavras em títulos e resumos foi realizada utilizando as seguintes entradas, em conjunto, vários ou disjunção: inovação tecnológica, tecnologia farmacêutica, classificação biofarmacêutica, doenças negligenciadas e indústria farmacêutica. Posteriormente, o conteúdo exposto foi analisado criteriosamente, levando em consideração os objetivos propostos para a realização da pesquisa.

\section{RESULTADOS E DISCUSSÃO}

A pobreza está indissociavelmente ligada a doenças infecciosas, incluindo a imunodeficiência humana infecção por vírus (HIV), tuberculose (TB), malária e doenças tropicais negligenciadas (DTNs). As DTNs compreendem infecções parasitárias, virais e bacterianas que afetam pessoas em todo o mundo, sendo as principais, priorizadas pela Organização Mundial da Saúde (OMS) a doença de chagas, leishmaniose, úlcera de buruli, cisticercose/teníase, filariose linfática, esquistossomose, helmintos transmitidos pelo solo (ascaris, ancilostomíase, chicote), dengue e raiva (HANSEN; PAINTSIL, 2016). Atualmente, existem muitos desafios associados à formulação de medicamentos pediátricos para países em 
desenvolvimento. Em particular, há uma falta de formas de dosagem aceitáveis pediátricas para a maioria das doenças tropicais negligenciadas, como a maioria das formas de dosagem oral é projetado para pacientes adultos. A população pediátrica, abrange de recém-nascidos a adolescentes (0-17 anos), é bastante heterogênea em relação a uma multiplicidade de fatores, incluindo a diferença nas habilidades de deglutição. (BALAN et al., 2017; BUCCIRECHTWEG et al., 2017; BUCKLEY et al., 2017; TRASTULLO et al., 2015).

O desenvolvimento de medicamentos pediátricos tem sido estimulado por recentes legislações (regulamentos dos EUA e da União Europeia), resultando em esforços significativos desenvolver formulações adequadas à idade aceitáveis para uso em pacientes pediátricos. Essas iniciativas, em conjunto com um processo mais sofisticado de descoberta de drogas e desenvolvimento, levaram a avanços significativos em à rotulagem de medicamentos para uso pediátrico (ELDER et al., 2017; BALAN et al., 2017; BUCCIRECHTWEG et al., 2017). Cápsulas ou comprimidos são as formas farmacêuticas de dosagem mais comum, mas também difíceis de engolir em pequenas crianças, especialmente entre as idades de 2 e 6 anos. Em 2007, quatro crianças com menos de 36 meses morreram de engasgamento em comprimidos de albendazol durante uma campanha de desparasitação na Etiópia. (TRASTULLO et al., 2015).

O cálculo da dose pediátrica é particularmente uma preocupação para os farmacêuticos. Outro desafio enfrentado pelos farmacêuticos é a indisponibilidade de formulações adequadas para uso pediátrico. Este também levou muitos farmacêuticos a preparar preparações líquidas extemporâneas, embora dados de estabilidade em tais preparativos são escassos. Algumas preparações extemporâneas contêm excipientes que são potencialmente prejudiciais em crianças (BALAN et al., 2017).

Um exemplo de fármaco para doenças negligenciadas é a ivermectina, indicado para o tratamento da filariose, onde só é aprovado o seu uso em adultos e crianças acima de 5 anos ou com mais de $15 \mathrm{~kg}$. A justificativa é devido à falta de dados clínicos suficientes para o uso, o que não estabelece a segurança e sua eficácia. Um outro exemplo é o praziquantel, utilizado para tratamento da esquistossomose. O mesmo só pode ser administrado em adultos e crianças acima de 4 anos, sendo justificado por falta de dados adicionais que assegurem a segurança do uso infantil com idade inferior a indicada. Além disso, o comprimido como forma farmacêutica para o praziquantel, dificulta o seu uso para esse público, uma vez que não pode ser partido ou mastigado (BRASIL, 2017a; BRASIL, 2017b).

As iniciativas de política pediátrica fizeram progresso significativo no avanço de um entendimento global de que a avaliação de novos medicamentos deve incluir formulações que atendam às necessidades pediátricas. Política e a reforma legislativa, iniciadas pelos Estados Unidos e União Europeia, levaram a um aumento no número de terapias avaliadas na população pediátrica. Os quadros regulatórios apropriados são um passo inicial vital para melhorar o desenvolvimento de medicamentos pediátricos (BUCCI-RECHTWEG et al., 2017; TSUKAMOTO et al., 2016). Diante do exposto, percebe-se a problemática relacionada a falta de medicamentos que englobe toda a classe pediatria. Diferentemente da ivermectina, há muitos trabalhos relacionados ao praziquantel para tratamento da esquistossomose destinados a esse público (BRASIL, 2017a).

\section{CONCLUSÃO}

As infecções parasitárias são uma das principais causas de morte e deficiência nas economias emergentes. Destina-se particularmente a populações vulneráveis, como crianças pequenas, e aqueles cujo sistema imunológico está comprometido por um estado nutricional 
fraco, anemia ou coinfecção com o vírus da imunodeficiência humana. Embora existam muitos medicamentos disponíveis, há uma necessidade urgente de novos. Todos os medicamentos utilizados contra doenças infecciosas correm o risco de desenvolver resistência. Além disso, há necessidade de novos medicamentos com regimes mais simples e que tenham melhor tolerabilidade e perfis de segurança destinados a administração pediátrica.

\section{REFERÊNCIAS}

OLIVEIRA LSS. As Doenças Negligenciadas e Nós [editorial]. Saúde Coletiva. 2009; 28:40-1.

EMA. Better Medicines for Children from Concept to Reality; 2013. Available at: http://ec.europa.eu/health/files/paediatrics/2013 com443/paediatric reportcom(2013)443 en.pdf. Accessed November 3, 2017

World Health Organization (WHO), 2007. Promoting safety of medicines for children. Pag 1-64. Available on line at: http://www.who.int/medicines/ publications/essentialmedicines/Promotion_safe_med_childrens.pdf. Accessed November 3, 2015.

BALAN, S.; HASSALI, M.A.; MAK, V.S.L. Challenges in pediatric drug use: A pharmacist point of view. Administrative Pharmacy, v. 13, p. 653-655, 2017.

BUCCI-RECHTWEG, C. Enhancing the Pediatric Drug Development Framework to Deliver Better Pediatric Therapies Tomorrow. Clinical Therapeutics, v. 1, p. 1-13, 2017.

BUCKLEY, L.A.; SALUNKE, S.; THOMPSON, K.; BAER, G.; FEGLEY, D.; TURNER, M.A. Challenges and strategies to facilitate formulation development of pediatric drug products: Safety qualification of excipients. International Journal of Pharmaceutics, v.1, p.1-7, 2017.

BRASIL. Agência de Vigilância Sanitária (ANVISA). Bulário eletrônico. Brainfarma Indústria Química e Farmacêutica S.A. 2017a. Disponível em: http://www.anvisa.gov.br/datavisa/fila_bula/frmVisualizarBula.asp?pNuTransacao $=4265612017 \& \mathrm{pI}$ dAnexo $=5378929$.

BRASIL. Agência de Vigilância Sanitária (ANVISA). Bulário eletrônico. Fundação Oswaldo Cruz. $2017 \mathrm{~b}$.

Disponível

em:http://www.anvisa.gov.br/datavisa/fila_bula/frmVisualizarBula.asp?pNuTransacao=2147362017 \&pIdAnexo $=4925944$

ELDER, D.P.; HOLM, R.; KUENTZ, M. Medicines for Pediatric Patients - Biopharmaceutical, Developmental, and Regulatory Considerations. Journal of Pharmaceutical Sciences, v.106, p.950960, 2017.

HANSEN, C.; PAINTSIL, E. Infectious Diseases of Poverty in Children A Tale of Two Worlds. Pediatr Clin N Am, v.62, p. 37-66, 2016.

TRASTULLO, R.; DOLCI, L.S.; PASSERINI, N.; ALBERTINI, B. Development of flexible and dispersible oral formulations containing praziquantel for potential schistosomiasis treatment of preschool age children. International Journal of Pharmaceutics, v.495, p.536-550, 2015.

TSUKAMOTO, K.; CARROLL, K.A.; ONISHI, T.; MATSUMARU, N.; BRASSEUR, D.; NAKAMURA, $H$. Improvement of Pediatric Drug Development: Regulatory and Practical Frameworks. Clinical Therapeutics, v. 38, p.574-581, 2016.

WELLS, T.N.C.; HOOFT VAN HUIJSDUIJNEN, R.; BURROWS, J.N. New Models of Drug Discovery and Development for Neglected Disease Populations. Comprehensive Medicinal Chemistry III, v.1, p. 491-504, 2017. 